\title{
Investigation of micrometastasis by cytokeratin staining in non-small cell lung cancer patients with tumor negative mediastinoscopic lymph node biopsies
}

\author{
Sinan Kaya ${ }^{a}$, Ahmet Başoğlu ${ }^{a}$, Yasemin Bilgin Büyükkarabacak ${ }^{a}$, Burçin Çelik ${ }^{a *}$, Ayşen Taslak Şengüla, \\ Tülin Durgun Yetim ${ }^{b}$, Levent Yıldız \\ ${ }^{a}$ Department of Thoracic Surgery, Ondokuz Mayis University Medical School, Samsun, Turkey \\ ${ }^{b}$ Department of Thoracic Surgery, Mustafa Kemal University Medical School, Hatay, Turkey \\ ${ }^{c}$ Department of Pathology, Ondokuz Mayis University Medical School, Samsun, Turkey
}

\section{ARTICLE INFO}

\section{Article History}

Received

$21 / 03 / 2013$

Accepted

$09 / 04 / 2013$

\section{* Correspondence to: \\ Burçin Çelik \\ Department of Thoracic Surgery, \\ Ondokuz Mayis University Medical School, \\ Samsun, Turkey \\ e-mail: cburcin@hotmail.com}

\section{Keywords:}

Cytokeratin

Immunohistochemical staining

Lung cancer

Mediastinoscopy

Micrometastasis

\section{ABSTRACT}

\begin{abstract}
To investigate micrometastasis by using epithelial cytokeratin marker in non-small cell lung cancer (NSCLC) patients with tumor negative mediastinoscopic lymph node biopsies. Micrometastasis were investigated by immunohistochemical staining in 20 patients who were diagnosed as NSCLC with ipsilateral mediastinal lymph nodes (RN2) in CT scan and with negative histopathologic mediastinoscopic lymph node biopsy (pN0). Frozen section was studied with hematoxylin-eosin (HE), a routine histopatological method in lymph nodes taken by mediastinoscopy and in absence of metastasis; resection was applied with thoracotomy in the same session. We studied micrometastasis in lymph nodes, which are taken out by mediastinoscopy and dissected during resection, using both HE and immunohistochemical staining with cytokeratin. All patients were male and median age was 60.9 (51-74) years. In 16 cases epidermoid carcinoma, in 2 cases adenocarcinoma and in 2 cases bronchioloalveoler carcinoma were diagnosed as histopathological types. Cervical mediastinoscopy was performed in 20 patients who were diagnosed as RN2 by thorax CT radiologically, and complete resection was done in pN0 patients. Sixty lymph nodes, 40 of them taken by mediastinoscopy and 20 taken during resection, were stained immunohistochemically in 20 NSCLC patients. Only one lymph node from a single patient was positive after staining. After postoperative staging, 18 patients were at a lower stage, one patient was at the same stage, and one patient with micrometastasis was at an advanced stage. Immunohistochemical staining may be useful in detecting micrometastasis in NSCLC. Standard cervical mediastinoscopy is still gold standard in preoperative staging. Efficiency can be increased by combining mediastinoscopy, a gold standard method in staging, with immunohistochemical methods.
\end{abstract}

J. Exp. Clin. Med., 2013; 30:119-124

C) 2013 OMU

\section{Introduction}

Lung cancer comes first among cancer originated deaths and is still characterized by first aggressive course and poor outcome. Despite curative resections, in early non-small cell lung cancer (NSCLC), recurrence is inevitable in one thirds of the patients and disease outcome is poorer than expected (Martini et al., 1995; Passlick and Pantel, 1996). This suggests that there may be micrometastasis which could not be detected by current clinical staging examinations. Conventional histopathological methods shows spreading to the regional lymph nodes or distant organs at the time of surgery. Recent advances in molecular biology lead to a better understanding in detection of micrometastasis (Chen et al., 1993; Gu et al.,
2002). In the past few years, several successful attempts have been made to detect micrometastasis in lymph nodes, bone marrow, and peripheral blood by either immunohistochemical staining or genetic methods (Chen et al., 1993; Izbicki et al., 1996; Passlick et al., 1996; Maruyama et al., 1997).

This study was designed to detect occult micrometastasis in non-small cell lung cancer patients with pN0 disease by epithelial cytokeratin (CK) staining, and to evaluate the relation between the micrometastasis in lymph nodes and the prognosis of the patients with completely resected.

\section{Material and methods}

In our clinic, we studied 20 patients prospectively, who are di- 
agnosed NSCLC and thought to have RN2 positive radiologically at the preoperative staging. They have been (between 2004-2005) applied the standard cervical mediastinoscopy and lymph node biopsy which is found to be histopathologically pN0. The stage of the disease based on the TNM classification of the revised International System for Staging for Lung Cancer (Mountain, 1997).

Cervical mediastinoscopy was performed in all patients before surgery and performed in 20 patients diagnosed as RN2 radiologically. Lymph node biopsy is taken from each patient by mediastinoscopy from two different stations and histopathologically evaluated using frozen section method. Patients who were staged as pN0 after mediastinoscopic biopsy underwent complete resection. Mediastinoscopy and resection were performed in the same session. Systemic lymph node dissection is applied during resection.

One of each upper mediastinal lymph nodes which were dissected from 20 patients who underwent complete resection and which were assessed as pN0 by hematoxylin-eosin (HE) staining was evaluated by cytokeratin stain. A total number of $60 \mathrm{pN0}$ lymph nodes were investigated. Two slices which were in $0.5 \mu \mathrm{m}$ thickness were prepared from each lymph node for immunohistochemical staining with polyclonal cytokeratin antibody, AE1/AE3 and monoclonal cytokeratin antibody C-8.

The following procedures were applied to the prepared specimens respectively; deparaffinization, incubation with the primary antibody (AE1/AE3, CK-8 antibody), incubation with the secondary antibody, fixation and staining with HE. All specimens were evaluated by the same pathologist (L.Y.) who had no knowledge of the patients clinical data and outcome. The presence of CK-positive cells within the body of the lymph nodes was considered to represent metastatic tumor cells even if only one single CK-positive cell was detected. After primary operation the patients were examined at 3-month intervals. Chest, abdominal and brain CT scans and bone scan were performed every 3 months. This study was approved by the ethics committee of the University.

\section{Statistical analysis}

The relationship between micrometastasis and clinicopathological characteristics ( $\mathrm{T}$ factor and histopathological type) was analyzed by Chi-square test. The patients have been divided into two groups; aggressive and non-aggressive. The sensitivity and specificity of the existence of micrometastasis in the aggressive and non-aggressive groups have been tested through the statistical diagnostic test. The statistical difference was considered to be significant if the $p$ value was below 0.05 .

\section{Results}

All patients were male and median age was 60.9 (51-74) years. Sixteen squamous cell carcinoma, 2 adenocarcinoma and 2 bronchioalveolar carcinomas were diagnosed histopathologically. Diagnosis was done with CT in 12 cases with transthoracic lung biopsy accompanied with CT and with bronchoscopic biopsy in 8 cases. After staging the localizations of tumor mass were as follows; 5 in the right upper lobe, 4 in the right middle lobe, 5 in the right lower lobe, 4 in the left upper lobe and 2 in the left lower lobe. Eight patients underwent lobectomy, 6 bilobectomy and 6 pneumonectomy.
The tumor localizations, histopathologic features and resection types are shown in Table 1.

\begin{tabular}{lcc} 
Table 1. Characteristics of the patients & & \\
Characteristics & N & \% \\
\hline Sex (M/F) & $20 / 0$ & $100 / 0$ \\
Localization of the tumor & & \\
Right upper lobe & 5 & 25 \\
Right lower lobe & 5 & 25 \\
Right middle lobe & 4 & 20 \\
Left upper lobe & 4 & 20 \\
Left lower lobe & 2 & 10 \\
Surgical Procedure & & \\
$\quad$ Lobectomy & 8 & 40 \\
Bilobectomy & 6 & 30 \\
Pneumonectomy & 6 & 30 \\
Histopathology & & \\
$\quad$ Squamous Cell Carcinoma & 16 & 80 \\
Adenocarcinoma & 2 & 10 \\
Bronchioalveoler Carcinoma & 2 & 10 \\
Diagnosis & & 60 \\
TTLB (Transthoracic Lung Biopsy) & 12 & 35 \\
Bronchoscopy & 7 & 5 \\
Exploratris thoracotomy & 1 & \\
\hline
\end{tabular}

A total number of 60 lymph nodes from 20 NSCLC patients were stained immunohistochemically. The regions where lymph nodes were taken and the ratio of pN0 lymph nodes which were examined by HE stain after mediastinoscopy are shown in Table 2.

\begin{tabular}{lcccccc}
$\begin{array}{l}\text { Table 2. Localizations of the mediastinoscopic and resectional } \\
\text { sampled lymph nodes }\end{array}$ \\
$\begin{array}{lccclcc}\text { Lymph nodes sampled by } \\
\text { mediastinoscopy }\end{array}$ & $\mathbf{N}$ & $\mathbf{\%}$ & $\begin{array}{l}\text { Lymph nodes } \\
\text { sampled by resection }\end{array}$ & $\mathbf{N}$ & $\%$ \\
\hline Upper paratracheal (No:2) & 14 & 35 & A-P Window (No:5) & 4 & 20 \\
Lower paratracheal (No:4) & 22 & 55 & Subcarinal (No:7) & 1 & 5 \\
Anterior subcarinal (No:7) & 4 & 10 & Paraesophageal (No:8) & 2 & 10 \\
& & & Hilar (No:10) & 2 & 10 \\
& & & Interlobar (No:11) & 7 & 35 \\
& & & Intralobar (No:12) & 4 & 20 \\
Total & 40 & 100 & & 20 & 100 \\
\hline
\end{tabular}

For each of the lymph nodes, both lower molecular weight (CK-8) and polyclonal CK (AE1/AE3) antibodies were stained, a total number of 120 specimens were examined. After immunohistochemical staining only one lymph node was found to be positive for micrometastasis. When the same node was examined by HE stain, there was no sign of any micrometastasis. After immunohistochemical staining a novel pathological staging was performed. The new stage of 18 patients was better than before whereas only the stage of one patient did not change and the one with micrometastasis was in an advanced stage. This patient received radiotherapy postoperatively, another patient with Stage IIIB (T4N0M0) received chemotherapy postoperatively. The clinical and pathological staging are shown in Table 3. The histologic type of carcinoma in which micrometastasis was detected by cytokeratin stain was squamous cell. The micrometastatic staining was seen in subcapsular region and medullary sinus of the lymph node. The microscopic views of the lymph node in which micrometastasis was detected are shown in Figure 1 and 2. 


\section{Table 3. Clinical and pathological staging}

\begin{tabular}{lcclcc} 
Clinical stage & $\mathbf{N}$ & $\mathbf{\%}$ & Pathological stage & $\mathbf{N}$ & $\mathbf{\%}$ \\
\hline T1N2M0 (IIIA) & 1 & 5 & T2N0M0 (IB) & 10 & 50 \\
T2N2M0 (IIIA) & 8 & 40 & T2N1M0 (IIA) & 3 & 15 \\
T3N2M0 (IIIA) & 10 & 50 & T3N0M0 (IIB) & 2 & 10 \\
T4N2M0 (IIIB) & 1 & 5 & T3N1M0 (IIB) & 3 & 15 \\
& & & T3N2M0 (IIIA)* & 1 & 5 \\
& & & T4N0M0 (III) & 1 & 5 \\
TOTAL & 20 & 100 & & 20 & 100 \\
\hline
\end{tabular}

*CK-positive cell detected by immunohistochemically staining in mediastinal LN

The relationship between $\mathrm{CK}$ positivity and $\mathrm{T}$ factor is compared by Fisher's chi-square test. When 20 patients were grouped according to $\mathrm{T}$ factor; 10 were $\mathrm{T} 3,8$ were $\mathrm{T} 2$, one was T4 and one was T1. Immunohistochemically, the stage of the patient who had micrometastasis was T3 (Table 4).

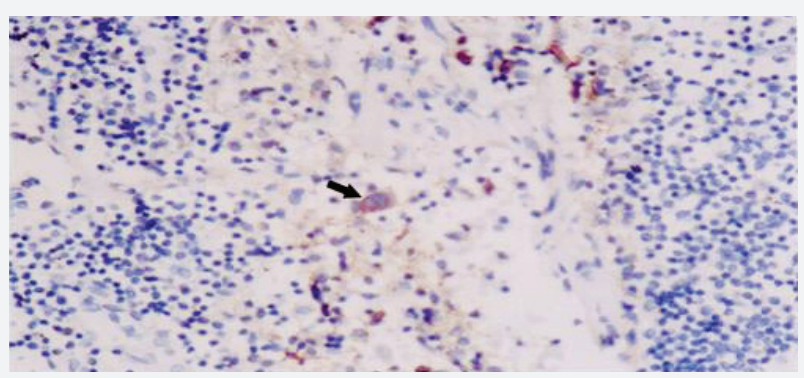

Fig. 1. A micrometastatic CK-positive (AE1/AE3) cell is seen in the sinus medullaris of the lymph node (HEX200).

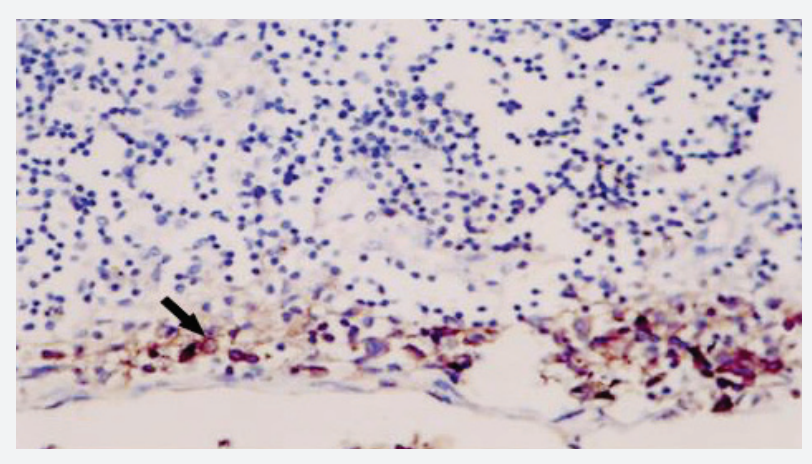

Fig. 2. A single micrometastatic CK-positive (AE1/AE3) cell is in the subcapsular region of the same lymph node (HEX200).

Table 4. Comparing of micrometastasis detecting by cytokeratin and $\mathrm{T}$ factor

\begin{tabular}{lcccc} 
Micrometastasis detecting by CK & \multicolumn{2}{c}{ T Factor } & Total & $\mathbf{p}$ \\
\hline & T3 & Others & & \\
Positive & 1 & 0 & 1 & \\
Negative & 9 & 10 & 19 & 0.250 \\
Total & 10 & 10 & 20 & \\
\hline
\end{tabular}

Micrometastasis could not be detected among 9 of 19 patients with CK staining. All 9 lesions were T3 and Chi square test revealed no statistical difference among the two groups.

Chi-square test was used to analyze the relationship Chisquare between the presence of micrometastasis and histologic subtypes. Four out of 20 patients had an aggressive course and early local recurrence together with distant metastasis was detected. Two of these patients had adenocarcinoma whereas the rest had squamous cell carcinoma, histopathologically. There was no statistically significant difference between the presence of micrometastasis and histopathological subtypes (Table 5). The cytokeratin stain was performed in both agressive and non-aggressive groups and the sensitivity, specificity and Youden's ratio were calculated as 25\%, \%100 and 0.25 , respectively (Table 6).

There was not any morbidity or mortality during the study period. Median hospitalization time was 11.65 (7-31) days.

\begin{tabular}{|c|c|c|c|c|}
\hline \multirow[t]{2}{*}{$\begin{array}{l}\text { Micrometastasis } \\
\text { detecting by } \mathrm{CK}\end{array}$} & \multicolumn{2}{|c|}{ Histopathology } & \multirow[t]{2}{*}{ Total } & \multirow[t]{2}{*}{$\mathbf{p}$} \\
\hline & $\begin{array}{c}\text { Squamous cell } \\
\text { carcinoma }\end{array}$ & Adenocarcinoma & & \\
\hline Positive & 1 & 0 & 1 & \\
\hline Negative & 1 & 2 & 3 & 1.00 \\
\hline Total & 2 & 2 & 4 & \\
\hline
\end{tabular}

Table 6. The values of sensitivity, specificity and Youden's ratio between the aggressive and Non-aggressive groups

\begin{tabular}{|c|c|c|c|}
\hline \multirow[t]{2}{*}{$\begin{array}{l}\text { Micrometastasis } \\
\text { detecting by CK }\end{array}$} & \multicolumn{2}{|c|}{ Course } & \multirow[t]{2}{*}{ Total } \\
\hline & $\begin{array}{c}\text { Agressive } \\
\text { Group }\end{array}$ & $\begin{array}{l}\text { Non-agressive } \\
\text { Group }\end{array}$ & \\
\hline Positive & 1 & 0 & 1 \\
\hline Negative & 3 & 16 & 19 \\
\hline \multirow[t]{2}{*}{ Total } & 4 & 16 & 20 \\
\hline & $\%$ & Lower limit & Upper limit \\
\hline Sensitivity & 25.00 & 0.00 & 67.43 \\
\hline Specificity & 100.00 & 100.00 & 100.00 \\
\hline Positive predicted $(+)$ value & 100.00 & 100.00 & 100.00 \\
\hline \multirow[t]{2}{*}{ Negative predicted (-) value } & 84.21 & 67.81 & 100.00 \\
\hline & Value & Lower limit & Upper limit \\
\hline Youden's Ratio & 0.25 & -0.1744 & 0.6744 \\
\hline
\end{tabular}

\section{Discussion}

Complete resection is the most preferred method for potential curative treatment in non-small cell lung cancer. Inspite of the curative treatment the outcome is still poor and the presence of lymph node metastasis is an important prognostic factor in lung cancer (Martini et al., 1995; Passlick and Pantel, 1996). Prognosis and effectiveness of treatment mostly depend on the stage of the disease in patients with NSCLC. Mediastinal lymph node metastasis has a negative impact on resectability and outcome (Luke et al., 1986).

Different methods are used in lung cancer staging and in evaluation of metastasis. Loco-regional lymph nodes and distant metastasis are evaluated by these methods. Non-invasive methods such as computed tomography (CT), magnetic resonance imaging (MRI) and positron emission tomography (PET) are routinely used together with invasive methods like mediastinoscopy, mediastinotomy, transbronchial fine-needle aspiration, endoscopic ultrasonography with fine-needle aspiration, video assisted thoracic surgery (VATS) and exploratris thoracotomy (Baron et al., 1982; Chen et al., 1993; Martini et al., 1995; Passlick and Pantel, 1996; Gu et al., 2002).

Computed tomography, which is the most preferred and very important diagnostic method especially in staging of lung cancer, is performed to assess the exact anatomic local- 
ization of the lesion and their local resectability. Despite high specificity (77-86\%), sensitivity (49-66\%) is low in evaluation of mediastinal lymph nodes with CT, (Brion et al., 1985; Toloza et al., 2003). Clinical data revealed that CT itself is not sufficient to identify mediastinal lymph node involvement in patients with NSCLC (Luke et al., 1986; Lowe et al., 1998; Schimmer et al., 2006). The main problem is the fact that CT only depicts the shape and size of mediastinal lymph nodes, rather than actual tumor involvement (Kramer and Groen, 2003).

During recent years PET has become a commonly used technique for the evaluation of mediastinal lymph nodes. In a study that compares PET and mediastinoscopy in the evaluation of mediastinal lymph node positivity, after mediastinoscopy lymph node metastasis was diagnosed in only 29 of 65 patients with positive mediastinal lymph node with PET, and false positivity rate was calculated as $55.4 \%$. On the other hand, after mediastinoscopy lymph node positivity was diagnosed in 16 patients out of 137 patients without positive node by PET and false negativity rate was reported as $11.7 \%$. PET specificity was $64.4 \%$ and sensitivity was $77.1 \%$ (GonzalezStawinski et al., 2003). In another study, Kernstine et al. demonstrated results 237 NSCLC patients. The N2 sensitivity was $80 \%$, the $\mathrm{N} 2$ specificity was $81 \%$. On the basis of these results, they concluded that PET provides helpful clinical information in patients with operable NSCLC or with a suspicious possibly operable NSCLC. PET does not eliminate the need for mediastinoscopy (Kernstine et al., 2002).

In potentially curable NSCLC patients, mediastinoscopy is still a reliable method which provides a thorough assessment of mediastinal involvement, predicts postoperative survival and prevents unnecessary thoracotomies (Gurses et al., 2002). The sensitivity of cervical mediastinoscopy was 67 $92 \%$ and specificity was $100 \%$ in evaluation of mediastinal lymph nodes (Toloza et al., 2003). In our study, cervical mediastinoscopy was performed in clinically staged twenty RN2 patients. Lymph node biopsy also performed in all patients during procedure. After histopathological evaluation twenty NSCLC patients with pN0 disease underwent complete resection.

Progress in molecular biology put forward new methods in the diagnosis of micrometastasis in lymph nodes. In patients to whom complete resection and systemic lymphadenectomy are applied, diagnosis of metastasis with immunohistochemical and genetic methods in lymph nodes where diagnosis was negative with routine histopathologic evaluation made the accuracy of preoperative staging controversial. One third of the patients who underwent radical surgical excision without distant or lymph node metastasis relapsed within 5 years during long term follow-up. These findings suggest that novel prognostic criteria should be added to the conventional TNM classification (International Breast Cancer Study Group 1990; Mountain, 1997).

The invasion of regional lymph nodes by small amounts of tumor cells was evaluated via serial dissection of several lymph nodes and staining them with HE accordingly. By using this method, the rate of micrometastasis detected in lymph nodes increased to $8-30 \%$. However, this method is not only time consuming but also unapplicable to daily practice. Instead, detection of micrometastasis by immunohistochemical staining which uses antibodies against epithelial tumor anti- gens seems to be more convenient.

In lung cancer, there are not many markers to be used in diagnosis of lymph node micrometastasis. Using markers like p53 and K-ras alone is not enough (Sugio et al., 1992; Traweek et al., 1993). In the last few years, cytokeratin is preferred as epithelial marker in the diagnosis of micrometastasis (Traweek et al., 1993; Nishio et al., 1996; Osaki et al., 2002). Micrometastasis are mostly found in the nodes closer to tumor bulk. So we tried to investigate micrometastasis in pN0 disease by using polyclonal and monoclonal antibodies (AE1/AE3, CK-8) in lymph nodes sampled by mediastinoscopy which is the gold standard in clinical staging. Micrometastasis detected by immunohistochemical staining is most commonly found in medullary sinuses in subcapsulary regions of the lymph nodes (Chen et al., 1993). In our study, micrometastasis are detected with CK in sinus medullaris and subcapsular region.

In a series of 49 patients having NSCLC at stage I pN0, $\mathrm{Gu}$ et al reported micrometastasis was diagnosed in $34.7 \%$ of the patients whom only $\mathrm{CK}$ is used, however, in patients whom p53 and CK is used together, rate of diagnosis increased to $44.9 \%$. In our study, we used only CK for immunohistochemical staining and only one out of 20 patients $(16 \%)$ had lymph node micrometastasis. The percentage of nodal micrometastasis of cytokeratin-positive cells has been reported between $10.4-70 \%$ in patients with lung cancer. Despite the patients in the series and the abundance of lymph nodes, there is a consensus on the necessity of larger and prospective studies about the diagnosis of micrometastasis (Chen et al., 1993; Izbicki et al., 1996; Passlick et al., 1996; Maruyama et al., 1997).

Our objective was to investigate micrometastasis by immunohistochemical staining in lymph nodes which were remote to the tumor localization and which may be potantially N2 or N3. In previous studies mostly hilar and lower mediastinal lymph nodes were evaluated. These lymph nodes are closer to the tumor mass situated in the lungs (Chen et al., 1993; Traweek et al., 1993). Age, gender, histopathological type, tumor size and the distance between tumor and lymph node were compared with the presence of micrometastasis and only the tumor size was found to be statistically significant (Chen et al., 1993).

In our study, paratracheal lymph node which was pN0 with HE by mediastinoscopy found to have micrometastatic with $\mathrm{CK}$. We grouped our patients into two as agressive and non-agressive postoperatively. In agressive group there were 4 patients. In one of these four patients local recurrence was seen five months after surgery and upper bilobectomy was converted to pneumonectomy. The pathological staging of the patient in whom micrometastasis with cytokeratin detected was T3N0M0. After immunohistochemical staining with CK the new pathological staging was T3N2M0. Thus, the patient had Stage IIB with routine histopathological methods but became Stage IIIA after cytokeratin staining. In this patient, vertebral metastasis was found three months after surgery. In other two patients, there were metastasis on the chest wall eight and ten months after surgery. In non-agressive group none of the 16 patients had micrometastasis with immunohistochemical method. These data confirmed that patients with micrometastasis have a poorer outcome compared to patients without micrometastasis. The survival of patients with occult 
metastasis was better than that of patients with overt metastasis and worse than that of patients with tumors confined to the lungs (Chen et al., 1993; Passlick et al., 1996).

The detection of micrometastasis in $\mathrm{pN} 0$ patients by mediastinoscopy is helpful for early systemic treatment. In patients who underwent complete resection and mediastinal lymph node dissection, the detection of micrometastasis by immunohistochemical methods may lead to the administration of adjuvant treatment due to the presence of the possible tumoral residue (Yasumoto et al., 2003).

All these data suggest that investigation of micrometastasis in mediastinal lymph nodes and distant organs should be considered in patients who have a surgically curable disease since relapses are likely in early NSCLC and survival can not be predicted accurately. However, there is still an ongoing debate about which methods and markers should be used. The results of the upcoming studies which will be carried out with larger patient series may be helpful in deciding the routine application of these methods in surgically curable patients.

In conclusion, the advances in molecular biology provides early detection of micrometastasis leading to the establishment of novel treatment and staging criteria in non-small cell lung cancer. The combination of mediastinoscopy, which is still the gold standard in staging, with immunohistochemical methods, may improve its efficacy.

\section{REFERENCES}

Baron, R.L., Levitt, R.G., Sagel, S.S., White, M.J., Roper, C.L., Marbarger, J.P., 1982. Computed tomography in the preoperative evaluation of bronchogenic carcinoma. Radiology. 145, 727-732.

Brion, J.P., Depauw, L., Kuhn, G., de Fracquen, P., Friberg, J., Rocmans, P., Struyven, J., 1985. Role of computed tomography and mediastinoscopy in preoperative staging of lung carcinoma. J. Comput. Assist. Tomogr. 9, 480-484.

Chen, Z.L., Perez, S., Holmes, E.C., Wang, H.J., Coulson, W.F., Wen, D.R., Cochran, A.J., 1993. Frequency and distribution of occult micrometastases in lymph nodes of patients with non-small-cell lung carcinoma. J. Natl. Cancer. Inst. 85, 493-498.

Gonzalez-Stawinski, G.V., Lemaire, A., Merchant, F., O’Halloran, E., Coleman, R.E., Harpole, D.H., D’Amico, T.A., 2003. A comparative analysis of positron emission tomography and mediastinoscopy in staging non-small cell lung cancer. J. Thorac. Cardiovasc. Surg. 126, 1900-1905.

Gu, C.D., Osaki, T., Oyama, T., Inoue, M., Kodate, M., Dobashi, K. Oka, T., Yasumuto, K., 2002. Detection of micrometastatic tumor cells in pN0 lymph nodes of patients with completely resected nonsmall cell lung cancer: Impact on recurrence and survival. Ann. Surg. 235, 133139.

Gurses, A., Turna, A., Bedirhan, M.A., Ozalp, T., Kocaturk, C., Demir, A., Ozcan, M., Urer N, 2002. The value of mediastinoscopy in preoperative evaluation of mediastinal involvement in non-small-cell lung cancer patients with clinical N0 disease. Thorac. Cardiovasc. Surg. 50, 174-177.

International Breast Cancer Study Group, 1990. Prognostic significance of occult axillary lymph node micrometastases from breast cancers. Lancet. 335, 1565-1568.

Izbicki, J.R., Passlick, B., Hosch, S.B., Kubuschock, B., Schneider, C., Busch, C., Knoefel, W.T., Thetter, O., Pantel, K., 1996. Mode of spread in the early phase of lymphatic metastasis in non-small-cell lung cancer: Significance of nodal micrometastasis. J. Thorac. Cardiovasc. Surg. $112,623-630$

Kernstine, K.H., Mclaughlin, K.A., Menda, Y., Rossi, N.P., Kahn, D.J., Bushnell, D.L., Graham, M.M, Brown, C.K., Madsen, M.T., 2002. Can FDG-PET reduce the need mediastinoscopy in potentially resectable nonsmall cell lung cancer? Ann. Thorac. Surg. 73, 394-402.

Kramer, H., Groen, H.J., 2003. Current concepts in the mediastinal lymph node staging of nonsmall cell lung cancer. Ann. Surg. 238, $180-188$.

Lowe, V.J., Fletcher, J.W., Gobar, L., Lawson, M., Kirchner, P., Valk, P., Karis, J., Hubner, K., Delbeke, D., Heiberg, E.V., Patz, E.F., Coleman, R.E., 1998. Prospective investigation of positron emission tomography in lung nodules. J. Clin. Oncol. 16, 1075-1084.

Luke, W.P., Pearson, F.G., Todd, T.R., Patterson, G.A., Cooper, J.D., 1986. Prospective evaluation of mediastinoscopy for assessment of carcinoma of the lung. J. Thorac. Cardiovasc. Surg. 91, 53-56.

Martini, N., Bains, M.S., Burt, M.E., Zakowski, M.F., McCormack, P., Rusch, V.W., Ginsberg, R.J., 1995. Incidence of local recurrence and second primary tumors in resected stage I lung cancer. J. Thorac. Cardiovasc. Surg. 109, 120-129.

Maruyama, R., Sugio, K., Mitsudomi, T., Saitoh, G., Ishida, T., Sugimachi, K., 1997. Relationship between early recurrence and micrometastases in the lymph nodes of patients with stage I non-small-cell lung cancer. J. Thorac. Cardiovasc. Surg. 114, 535-543.

Mountain, C.F. 1997. Revisions in the International System for Staging Lung Cancer. Chest. 111, 1710-1717.

Nishio, M., Koshikawa, T., Kuroishi, T., Suyama, M., Uchida, K., Takagi, Y., Washimi, O., Sugiura, T., Ariyoshi, Y., Takahashi, T., Ueda, R., Takahashi, T., 1996. Prognostic significance of abnormal p53 accumulation in primary, resected non-small-cell lung cancers. J. Clin. Oncol. 14, 497-502.

Osaki, T., Oyama, T., Gu, C.D., Yamashita, T., So, T., Takenoyama, M., Sugio, K., Yasumoto, K., 2002. Prognostic impact of micrometastatic tumor cells in the lymph nodes and bone marrow of patients with completely resected stage I non-small-cell lung cancer. J. Clin. Oncol. 20, 2930-2936.

Passlick, B., Pantel, K., 1996. Prognostic factors in stage I non-small cell bronchial carcinoma. Zentralbl. Chir. 121, 851-870.

Passlick, B., Izbicki, J.R., Kubuschok, B., Thetter, O., Pantel, K., 1996. Detection of disseminated lung cancer cells in lymph nodes: Impact on staging and prognosis. Ann. Thorac. Surg. 61, 177-183.

Schimmer, C., Neukam, K., Elert, O., 2006. Staging of non-small cell lung cancer: Clinical value of positron emission tomography and mediastinoscopy. Interact. Cardiovasc. Thorac. Surg. 5, 418-423.

Sugio, K., Ishida, T., Yokoyama, H., Inoue, T., Sugimachi, K., Sasazuki, T., 1992. Ras gene mutations as a prognostic marker in adenocarcinoma of the human lung without lymph node metastasis. Cancer. Res. 52, 2093-2096.

Toloza, E.M., Harpole, L., McCrory, D.C., 2003. Noninvasive staging of non-small cell lung cancer: A review of the current evidence. Chest. $123,137-146$

Toloza, E.M., Harpole, L., Detterbeck, F., McCrory, D.C., 2003. Invasive staging of non-small cell lung cancer: A review of the current evidence. Chest. 123, 157-166. 
Traweek, S.T., Liu, J., Battifora, H., 1993. Keratin gene expression in non-epithelial tissues. Detection with polymerase chain reaction. A.M. J. Pathol. 142, 1111-1118.

Yasumoto, K., Osaki, T., Watanabe, Y., Kato, H., Yoshimura, T., 2003. Prognostic value of cytokeratin-positive cells in the bone marrow and lymph nodes of patients with resected nonsmall cell lung cancer: a multicenter prospective study. Ann. Thorac. Surg. 76, $194-202$. 